\title{
Using color photometry to separate transiting exoplanets from false positives
}

\author{
B. Tingley \\ Research School of Astronomy and Astrophysics, ANU Cotter Road, Weston, Canberra ACT 2611, Australia \\ e-mail: tingley@mso.anu.edu.au
}

Received 3 December 2003 / Accepted 2 June 2004

\begin{abstract}
The radial velocity technique is currently used to classify transiting objects. While capable of identifying grazing binary eclipses, this technique cannot reliably identify blends, a chance overlap of a faint background eclipsing binary with an ordinary foreground star. Blends generally have no observable radial velocity shifts, as the foreground star is brighter by several magnitudes and therefore dominates the spectrum, but their combined light can produce events that closely resemble those produced by transiting exoplanets.

The radial velocity technique takes advantage of the mass difference between planets and stars to classify exoplanet candidates. However, the existence of blends renders this difference an unreliable discriminator. Another difference must therefore be utilized for this classification - the physical size of the transiting body. Due to the dependence of limb darkening on color, planets and stars produce subtly different transit shapes. These differences can be relatively weak, little more than $1 / 10$ th the transit depth. However, the presence of even small color differences between the individual components of the blend increases this difference. This paper shows that this color difference is capable of discriminating between exoplanets and blends reliably, theoretically capable of classifying even terrestrial-class transits, unlike the radial velocity technique.
\end{abstract}

Key words. stars: planetary systems - occultations - methods: data analysis

\section{Introduction}

Of all of the difficulties facing the search for transiting exoplanets, the issue of certainty is hardest to resolve. There are many phenomena capable of producing events that appear very similar to planetary transits. It is an observational challenge to separate the exoplanets from "false positives" - the non-planetary sources that manifest low-amplitude transit-like events. Currently, radial velocities are measured for exoplanetary candidates to make this discrimination. These measurements are capable of identifying grazing eclipsing binaries, which are the primary source of false positives. Beyond this, however, they are of limited use. They cannot, for example, classify exoplanets significantly smaller than Jupiter - currently, the least massive exoplanet discovered by the radial velocity technique is $0.2 M_{\mathrm{J}}$. This limitation is caused by the natural asteroseismic radial velocity oscillations present in main sequence stars, which mask the radial velocity shifts. Moreover, the need for ultra high-resolution spectra to detect the radial velocity shifts limits the depth to which exoplanets can be verified. Even the best facilities in the world can only go so deep before it becomes impossible to attain the necessary spectral resolution to identify even short-period Jovian planets. These effects have already impacted efforts to discover exoplanets and will be a major obstacle for ambitious projects such as Kepler and COROT, which aim to discover terrestrial exoplanets. Without any means to classify the vast numbers of very shallow transiting systems consistent with exoplanets that are significantly less than a Jupiter mass, it will be difficult to achieve the science goals of the missions, to identify planets and estimate the fraction of stars with planets.

However, the most serious failing of radial velocities is their inability (in most cases) to identify "blends", a chance superposition where the light from a star is contaminated by that of a fainter eclipsing binary along the same line of sight. In this circumstance, the depth of the eclipse is reduced by the presence of the bright ordinary star, possibly to the point that it is consistent with an exoplanetary transit. To compound this problem, the combined light of a blend is often dominated by the brighter star, hiding the radial velocity shift of the faint binary. In some circumstances, high-resolution spectra can reveal blends through the presence of strong line asymmetries or extra spectral lines rather than radial velocity variations (Konacki et al. 2003). Another recently published technique proposes using observed transit parameters to identify blends, effectively taking advantage of the change in shape of the transit between blends and exoplanets (due to limb darkening and size differences) to make the identification (Seager \& Mallén-Ornelas 2003). It can be difficult to measure the shape of the transit accurately enough for classification purposes, however.

The OGLE-III campaign (Udalski et al. 2002a,b), the most successful planet hunt to date, demonstrated the various 
limitations of the radial velocity technique. Of the 59 candidates that were identified during the 2001 campaign of OGLEIII, 7 were considered too faint for follow-up observation and only 6 of the remaining candidates had radial velocity variations less than a few $\mathrm{km} \mathrm{s}^{-1}$ and no sign of secondary transits or out-of-transit variations. Of these 6 , one was clearly a blend, one clearly a planet, and the other 4 remain unclassified. This means that out of the 46 that were truly interesting candidates, 11 could not be classified using radial velocities, despite the use of the world-class facilities Keck and VLT to make the observations (Konacki et al. 2003; Dreizler et al. 2003).

Clearly, the radial velocity technique will be insufficient for the needs of satellites such as Kepler and COROT. These projects will not go as deep as OGLE-III, but can be expected to observe a large number of transit-like events of all depths, both Jovian-class (corresponding to a giant planet) and terrestrialclass (corresponding to a terrestrial planet). Clearly, the success of these projects is crucially dependent on the ability to classify exoplanet candidates reliably.

Another method for classifying transits has appeared many times in the literature over the past 35 years but has never received a full development. It was first mentioned by Rosenblatt (1971), then again some years later by Borucki et al. (1984), and recently by Drake (2003) and Charbonneau (2003), who is involved in a group assembling a telescope dedicated to the use of this technique for classifying planet candidates (Kotredes et al. 2003). It involves observing the color change of the system during transit - most exoplanet transits produce a very distinctive color index "signature" that is clearly distinguishable from a grazing binary or a blend. The strength of this signature in $B-I$ is about $15 \%$ of the depth of the transit in the individual colors and will usually be even stronger for blends. Only in the unlikely occurrence that all three stars in the blend have essentially the same $B-I$ will a blend have a signature weaker than this.

These differences between exoplanet transits and binary or blended transit are potentially of great import in the search for exoplanets, allowing for a better classification of candidates. The sensitivity of blended transits to color difference only increases its utility. This article will explore these differences and demonstrate their practicality. First, the method for modeling the transits and constructing the blends will be discussed. The $\delta(B-I)$ and $\delta(V-I)$ of exoplanets and blends will then be compared, exploring differences in color between the components. The results will demonstrate that this method is a viable technique for discriminating between blends and exoplanets.

\section{Modeling the transits and constructing blends}

Modeling transits is a basic application of generic limb darkening laws. Complex limb darkening laws exist, but the degree to which they match reality is poorly known. It is an observational challenge to measure limb-darkening, especially for solar-type (and later) stars. The limb darkening for only one such star (besides the Sun) has been observed, from a high-magnification microlensing event (Abe et al. 2003). In addition, metallicity has an effect on limb darkening and is in most cases an unknown. For these reasons, it is sufficient for the purposes of this analysis to use a simple limb darkening law, especially as only the lower main sequence is of concern and only first order precision can be expected regardless. The limb darkening law used, taken from Astrophysical Quantities (Allen 1976), is

$\frac{I_{\lambda}^{\prime}(\theta)}{I_{0}^{\prime}(\theta)}=1-u_{1}(1-\cos \theta)$

where $u_{1}$ is estimated for the $B, V$ and $I$ filters, using Astrophysical Quantities and weighted for the passbands from Bessell (1990). The constants derived are: 0.75 for $B, 0.55$ for $V$ and 0.4 for $I$. Of course, these values are for the Sun and there are inevitably changes along the main sequence. However, the characteristic of this limb darkening law that makes this technique possible is preserved - the increased concentration of blue light to the center of the disk compared to the red light, evidenced by the fact that $u_{1}$ is almost twice as large for $B$ as it is for $I$.

To construct a blend, one must take the results from a modeled binary eclipse and its colors and combines them with a third star with the proper separation to produce a blended transit with the desired depth in the chosen color. A blend effectively consists of three components that affect the color change during transit: a constant component and two eclipsing components. The constant component can exist of any number of stars along the line of sight, and can be physically in front of or behind the eclipsing components - or even both. Ultimately, it is only the colors and magnitude of the constant component and the eclipsing components, plus the depth of the unblended eclipse in the various colors and the separation of the components that matter. This method of constructing blends is otherwise completely general.

A relationship for determining the proper separation can be derived from the modeled depth of the binary eclipse and the desired depth of the blend using the basic relation between magnitudes and fluxes:

$m_{1}-m_{2}=-2.5 \log \frac{f_{1}}{f_{2}}$

where $m_{1}$ and $m_{2}$ are the magnitudes of the two components to be compared and $f_{1}$ and $f_{2}$ are their corresponding fluxes. This equation is then applied twice: once to the blend outside eclipse and at eclipse maximum and again to the constant component and the eclipsing component outside of eclipse. With this, the following equation can be derived:

$m_{\mathrm{e}}=m_{\mathrm{c}}-2.5 \log \left(\frac{1-10^{-0.4 D}}{10^{-0.4 D}-d}\right)$

where $m_{\mathrm{e}}$ is the total magnitude of the eclipsing component outside of transit, $m_{\mathrm{c}}$ is the magnitude of the constant component, $D$ is the desired depth of the blended transit in magnitudes and $d$ is the fractional depth of the binary eclipse. This can be extended for magnitudes in a single filter, but then the transit depths in that filter must be used.

\section{Analysis}

The analysis in this paper uses transit depths of the blends in $I$ and considers two different classes transit depths, Jovian 


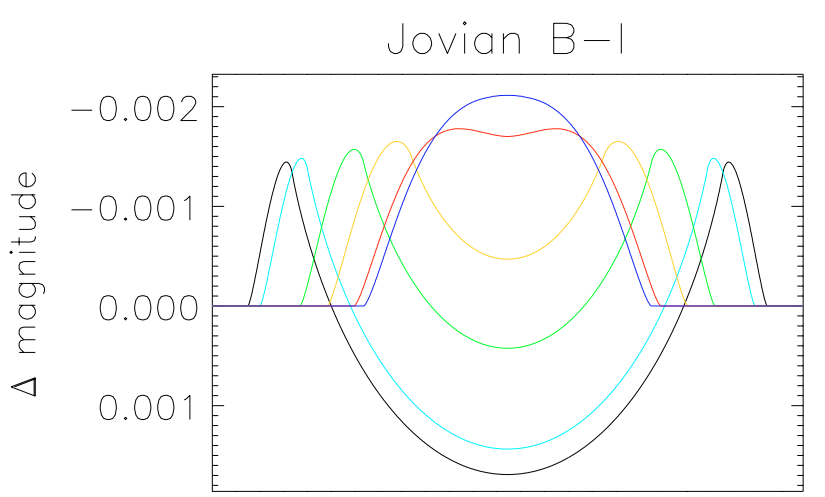

time

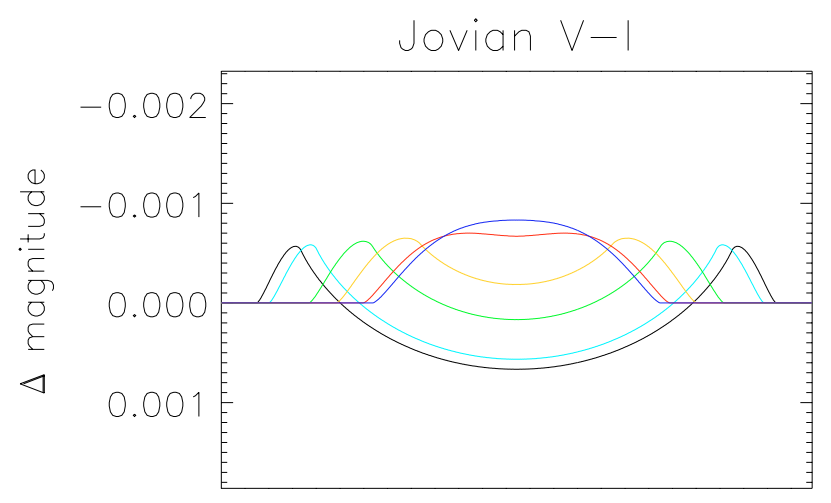

time

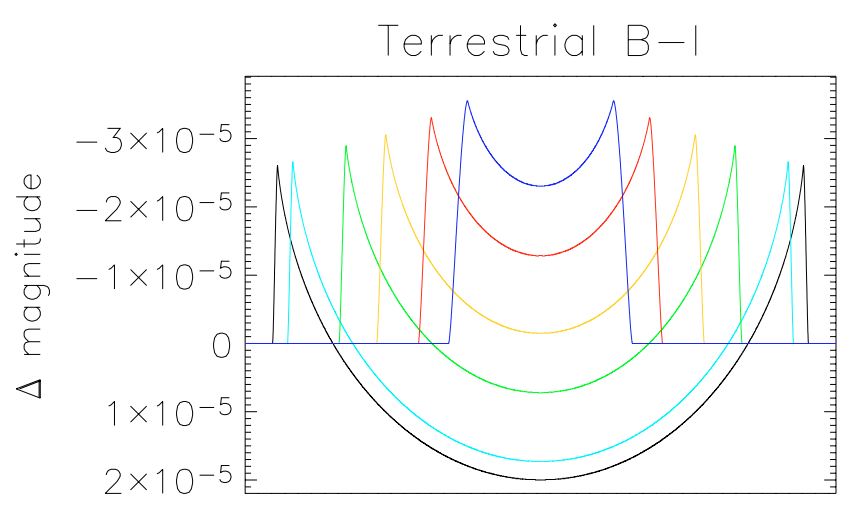

time

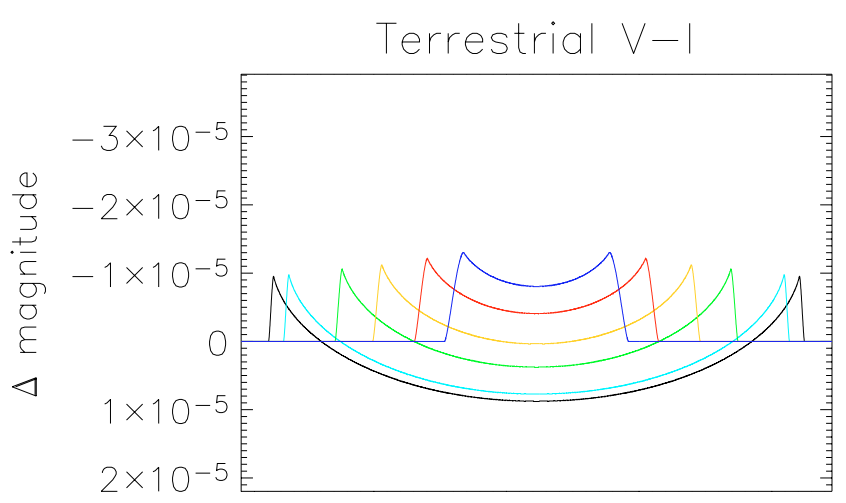

time

Fig. 1. Modeled Jovian (depth $=0.01 \mathrm{mag}$ in $I$ ) and terrestrial (depth $=0.0001 \mathrm{mag}$ ) transits for different projected inclinations in both $B-I$ and $V-I$. The projected inclinations used were 0 (broadest), $\frac{R}{3}, \frac{2 R}{3}, \frac{4 R}{5}, \frac{9 R}{10}$ and $\frac{19 R}{20}$ (narrowest). The double-horned profile is typical of exoplanetary transits - stellar blends cannot produce it. However, grazing Jovian transits without the double-horned profile can be mimicked by blends. The size of the transiting planet must be increased for increasing projected inclinations to maintain a transit depth in $I$.

(depth $=0.01 \mathrm{mag})$ and terrestrial (depth $=0.0001 \mathrm{mag})$. It explores the effect of color differences between the constant component and the eclipsing components. It also explores differences in color between the eclipsing components. The most likely case where all three components of the blend will be different colors is a logical extension of the studied cases. Beyond stellar blends, there is the possibility that a transiting Jovian exoplanet might blend with other stars to create a terrestrial-class transit. The effects of color on this hypothetical blend are also explored.

Due to the nature of the analysis, it is only the color differences between the components that is significant. The actual absolute colors of the components involved is irrelevant. So, while a spectral type of G2 was used to make the figures, any spectral type could have been used and the results would have been the same, aside from minor differences in the limb darkening laws and radii of exoplanets used in order to model transit depths.

\section{Results of transit analysis}

Figure 1 shows the $B-I$ and $V-I$ for Jovian and terrestrial exoplanets at different projected inclinations, defined as the closest approach of the center of the secondary to the center of the primary during transit. The sizes of the modeled planets were adjusted slightly so that the transits would have the same depth in $I-0.01$ mag for Jovian planets and $0.0001 \mathrm{mag}$ for terrestrial.

Both terrestrial and Jovian exoplanets create transits which have double horned profiles for most projected inclinations. These horns are more than twice as high in $B-I$ as they are in $V-I$ and are sharper for terrestrial exoplanets than they are for Jovian. They occur because of the small size of the exoplanets compared to the size of the star, which accentuates the general property of limb darkening that photospheres are more centrally concentrated at shorter wavelengths. This means that as the exoplanet crosses the limb, the star appears more blue, as red light is preferentially occulted. As the exoplanet moves across the disk of the star, the star appears more red as the exoplanet completely clears the limb of the star (allowing the occulted red light in again) and begins preferentially occulting blue light.

In Fig. 1, the height of these horns appears to increase with projected inclination. This is however a result of the fact that these transits are modeled to have a fixed depth in $I$. Maintaining this requires an increase in exoplanet radius, as transits away from central are not as strong, which in turn 

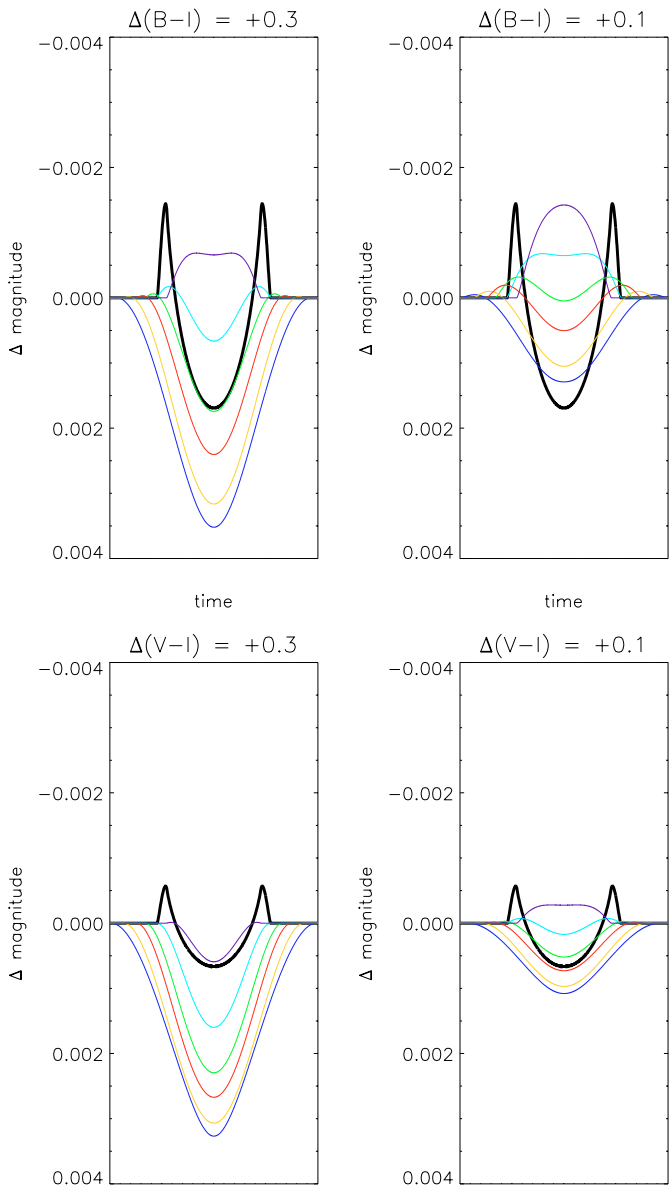

time

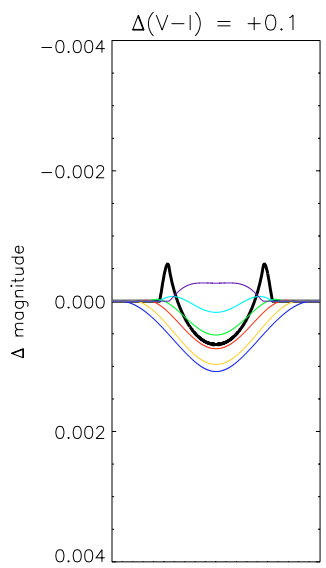

time
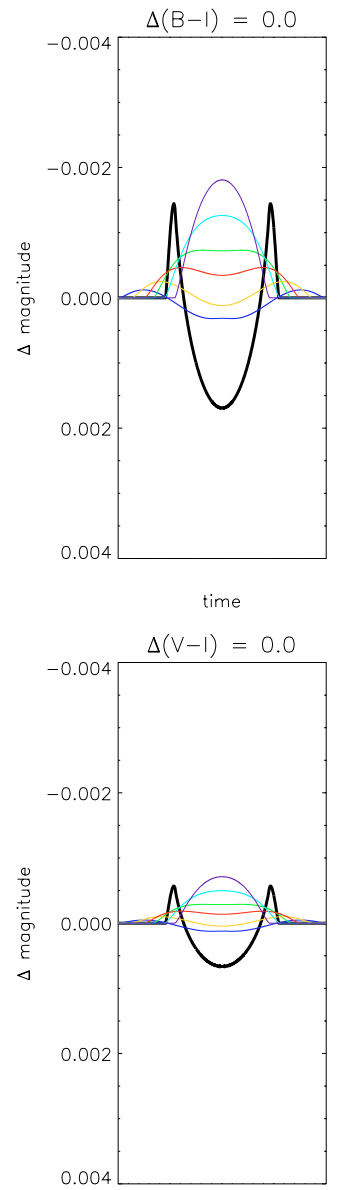

time
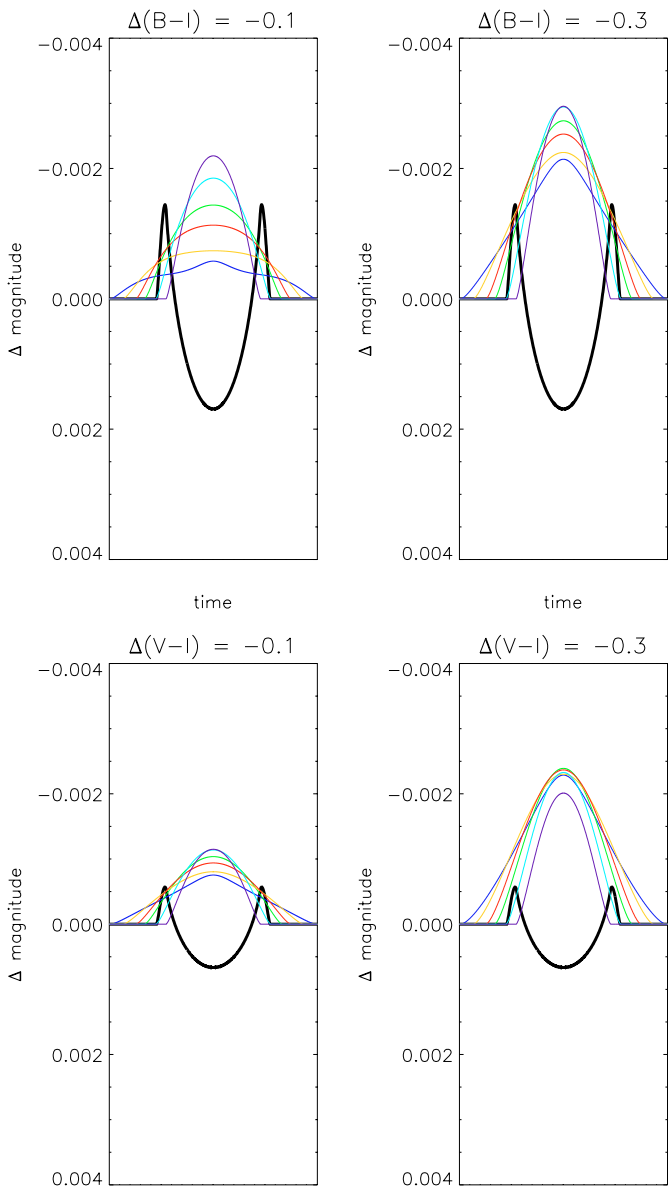

time

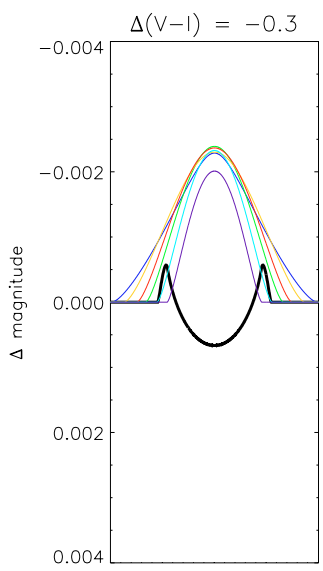

time

Fig. 2. Blended Jovian-class transits in instances where the constant component has different colors than the eclipsing components, which are identical. The thin lines depict different projected inclinations for the given color difference. The projected inclinations used are $\frac{R}{3}$ (broadest), $R, \frac{4 R}{3}, \frac{3 R}{2}, \frac{5 R}{3}$ and $1.78 R$ (narrowest). The heavy black line is a central $(i=0)$ Jovian transit shown for comparison. Further diluting the light to form a terrestrial-class blend will not change the shape of the blended events, only their depth, so the corresponding modeled terrestrial-class events are not included.

increases the height of the horns. If the exoplanet radius had been fixed, the horns would be the same height for all projected inclinations where the exoplanet crosses the limb of the star entirely, as that is that point where the horn occurs. Obviously, the horn should have the same height under these conditions, as it occurs the same exoplanet/star configuration - the exoplanet lying just over the limb of the star.

Figure 2 shows a series of plots that depict the $B-I$ and $V-I$ of blends constructed of identical eclipsing components with constant components of slightly different colors for several different projected inclinations. For these blends, the separation between the eclipsing and constant components was adjusted such that there was a fixed transit depth in the combined emission, as described in Eq. (2). It is important to realize that changes in $B-I$ and $V-I$ can be treated independently - the shape of the transits in $B-I$ is set by the $\Delta(B-I)$ between the constant and eclipsing components and independent of any differences in any other color band.

The thick line superimposed over these plots is a modeled central Jovian transit of the same depth. By comparing Figs. 1 and 2, one can see the the worst case scenarios involve grazing
Jovian transits, which lack a double-horned profile. It is possible to construct a blend in this case that would mimic a giant planet to the point that they would be indistinguishable. However, it would require that the eclipsing component had the same $V-I$ as the constant component while being slightly bluer (the $\Delta(B-I)$ s shown is Fig. 2 are for the constant component) and moreover be at least moderately grazing. This is helpful, as grazing eclipses are swallower, requiring that that the separation between the two components must be reduced to compensate. As the density of background stars increases rapidly with magnitude, this decreases the probability that a configuration that produces a Jovian transit-like event could occur while increasing the chance that its nature might be betrayed by spectra taken for radial velocity measurements.

The corresponding figures for terrestrial-class blends are not shown. Blended events of this depth are virtually identical in shape to those shown in Fig. 2 for blended Jovian-class events, except that they are scaled down by a factor of approximately 100 . Moreover, terrestrial exoplanets will very nearly always cross the limb of the star entirely if they transit at all, as they are much smaller than stars. Strictly from geometrical 

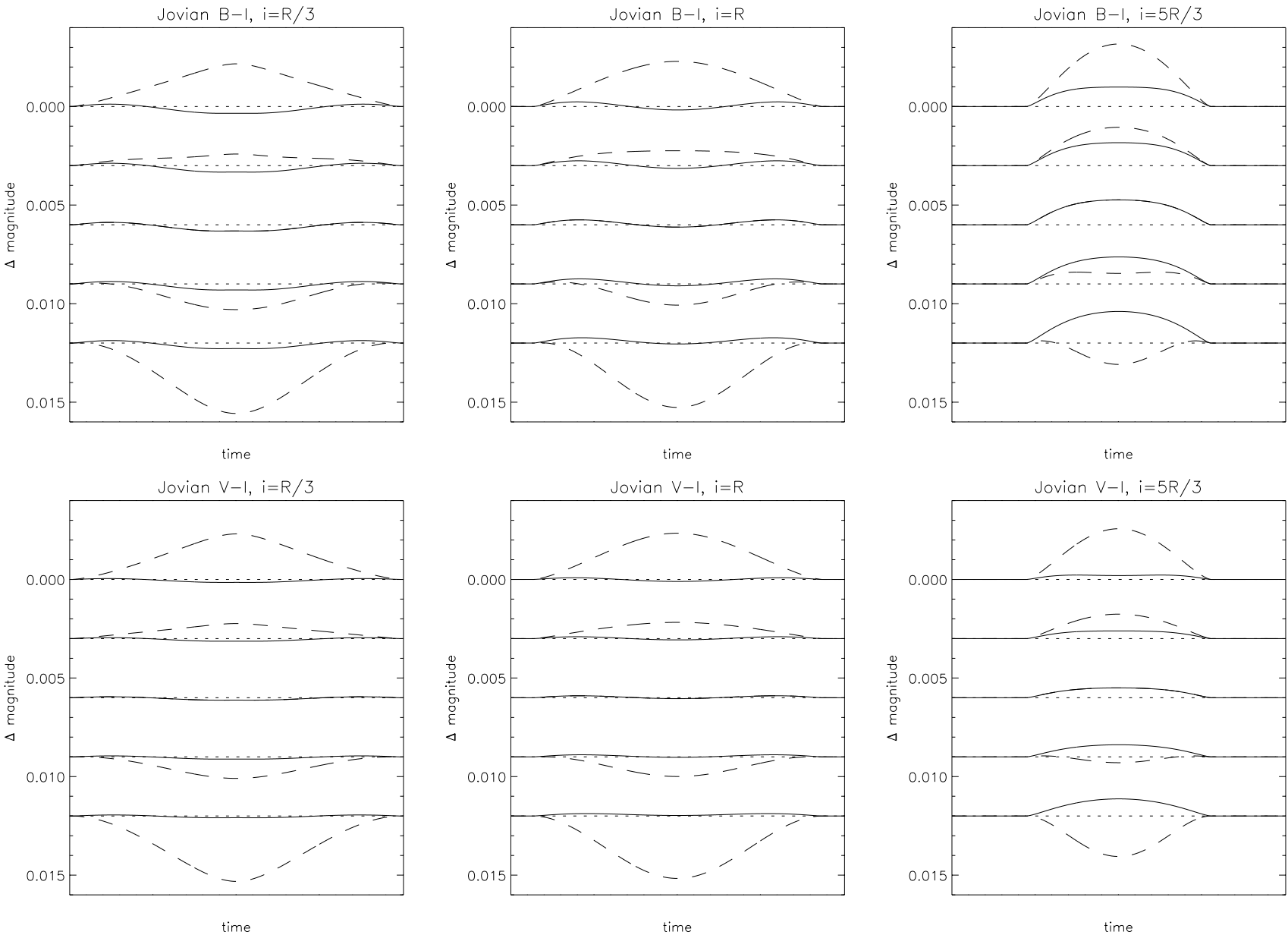

Fig. 3. The primary and secondary transits for the case where one of the the eclipsing components is different from the other two components in the blend for different projected inclinations (designed with $i$ ) and color differences. The top line in each figure is color +0.3 , followed by $+0.1,+0.0,-0.1$ and down to -0.3 on the bottom. The primary eclipse is represented by a dashed line while the secondary is a solid line.

arguments, terrestrial planets, which have a radius approximately $1 \%$ that of a typical star, would completely cross the limb of the star approximately $98 \%$ of all transiting cases. Most likely these extreme events would not be detected at all, as they would not last very long and would have a very low amplitude. As such, they will be left out of the blend analysis for the sake of brevity - except in the potentially interesting case of background transiting Jovian exoplanet blends.

Figure 3 explores differences caused by changing the color of one of the stars in the eclipsing component, showing the primary and second transits in $\Delta(B-I)$ and $\Delta(V-I)$ for three different projected inclinations and a variety of color changes. These figures clearly show that altering the binary pair significantly changes the primary and secondary transits. Obviously, an exoplanet only produces one transit per orbit, which will always look the same. This effect can therefore be used to differentiate between exoplanets and blends, although color differences again appear to be the dominant variable. Another potential variable, the size difference between the stars in the eclipsing components, was also explored but found to have very little impact for reasonable (10\%) changes.

Another possibility that should be examined is one where a background transiting Jovian exoplanet is the eclipsing component of a blend. When combined with a relatively bright constant component, the depth of the transit of a Jovian exoplanet can be reduced to the point that it is appears terrestrial in origin. Satellite projects such as Corot and Kepler will have several tens of thousands of target stars, generally between magnitudes 10 and 14 . While only a fraction of these target stars will have background stars of the type that could potentially have exoplanets - a quick examination of an OGLE-III bulge field (Udalski et al. 2002c) suggests that only about 1 out of 60 of the stars between magnitudes 14 and 14.5 will have a background star that is two to four magnitudes fainter within an arcsecond. A true blend requires a separation much less than an arcsecond, so it seems extremely unlikely that this sort of blend would occur. However, the possibility remains, so an analysis is in order.

Figure 4 depicts transiting Jovian planet blends for several projected inclinations and color differences. Despite the fact that Jovian exoplanetary blends can produce double-horned profiles, only in the case of a central Jovian transit will the blended events resemble terrestrial transits - and then only when the color difference between the constant component and the exlipsing component is very small (less than 0.1). Even small color changes have a strong effect on the shape of the blended events. 


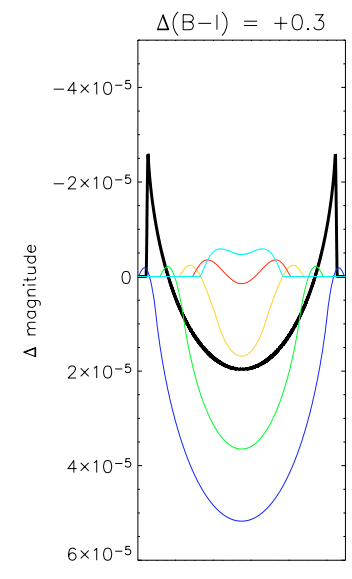

time

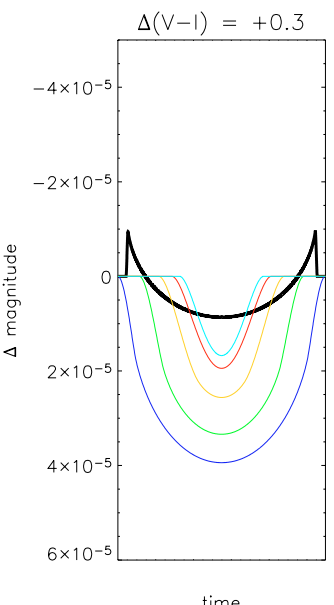

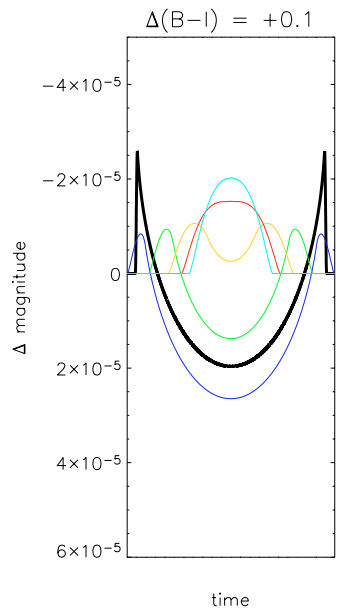

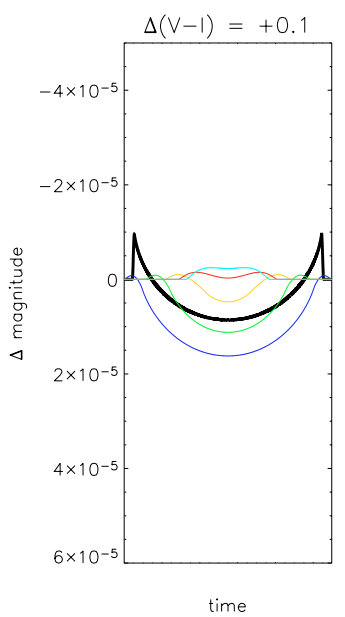

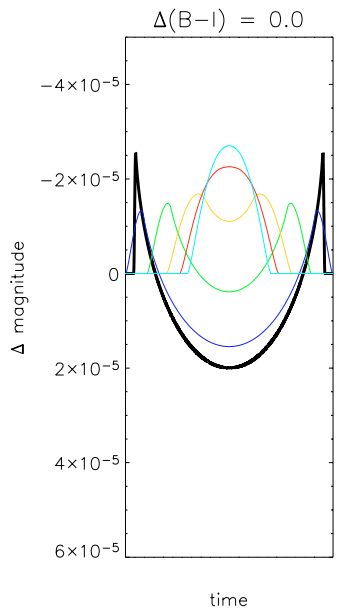

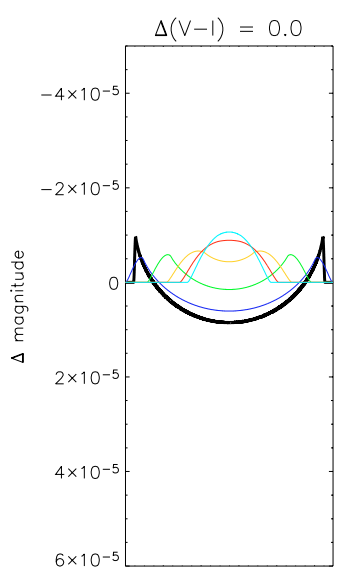

time
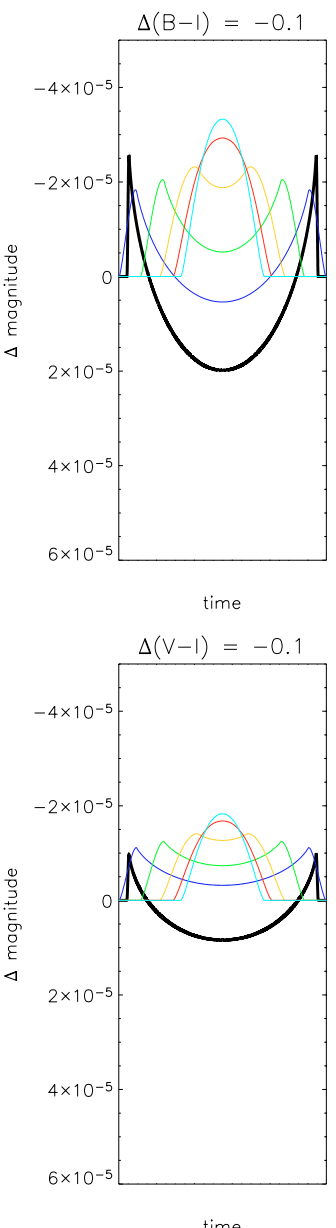
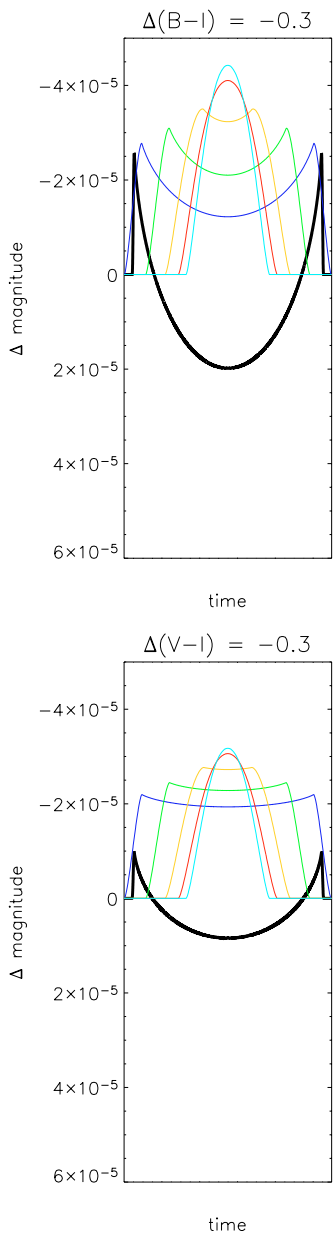

Fig. 4. The effect of color change on transiting Jovian exoplanets blended with a constant component to form a terrestrial-class event. The thick solid line is a central terrestrial transit, while the thin lines are the blended events with different projected inclinations -0 (broadest), $\frac{2 R}{3}, 0.87 R$, $0.96 R$ and $R$ (narrowest). Central Jovian blends can resemble terrestrial transits, but even small color changes between the eclipsing component and the constant component in the blend have a strong effect on the blended events.

\section{Conclusions}

The color index of transits is a useful discriminator between exoplanets and blends. Non-grazing exoplanet transits exhibit a double-horned profile that is distinct from the bell-curves produced by blends. As the limits of this method are technical (instrumental precision) rather than intrinsic (asteroseismic oscillations in radial velocity), it can be used on exoplanet candidates that lack an observerable radial velocity signal due to low exoplanet mass, large orbital radius and/or high magnitude. Moreover, any color difference between the components of a blend increases the strength of the blend signature. This, coupled with the likelihood that the fainter eclipsing component will be redder than the static component in most cases (either because it is a foreground M-dwarf pair or because it lies in the background and therefore subject to more extinction than the static component), means that the signature from a blend will often be stronger than the signal from an exoplanet, making them easier to identify.

There are only a few cases where this technique fails. Grazing exoplanet transits fail to produce distinctive doublehorned signatures - only about $20 \%$ of all Jovian exoplanets and about $2 \%$ of all terrestrial exoplanets will cause grazing transits. Even in these cases, the color change in $B-I$ is twice that as in $V-I$, a characteristic not likely to be produced by a blend, as it requires the color differences between the static component and the eclipsing component $(\Delta(B-I)$ and $\Delta(V-I))$ to be within approximately 0.05 of each other. This is a relatively unlikely scenario in the presence of extinction.

For Jovian-class events, it is difficult but not impossible to obtain the precision needed to observe the difference between blends and exoplanets from the ground. A Jovian exoplanet would produce a signature with a strength in excess of $1 \mathrm{mmag}$, which is approximately the limit for ground-based observations in a single color magnitude (see, for example, Gilliland et al. 1993). Creating a color from two color magnitudes decreases the precision, but the relatively long duration of the events allows for it to be observed many times each transit and their periodic nature allows for the transit to be observed multiple times. This makes is possible to build up the necessary statistics to enable a credible classification.

It is far more difficult to classify terrestrial-class events. Their expected signature would be around $10 \mathrm{ppm}$, which is not possible with any current instrument. The HST observations of the transiting exoplanet in HD 209458 reached a 
precision of $110 \mathrm{ppm}$, for example (Brown et al. 2001), using absolute photometry. Instruments designed specifically for precision photometry can do better. One example of this is the Kepler mission, which expects a noise level of approximate $74 \mathrm{ppm}$ at the 15 min level for a G2 star with $m_{R}=12$ (Jenkins \& Doyle 2003). Another, the recently-cancelled MONS mission, was specifically designed for precision two-color photometry of a single target star. It expected to reach a noise level of about $54 \mathrm{ppm}$ at the $15 \mathrm{~min}$ level in color intensity ratio - equivalent to color - for a star with $m_{V}=5$ (Christensen-Dalsgaard 2002). That means that a precision of $10 \mathrm{ppm}$ could be reached in about $13.7 \mathrm{~h}$ for Kepler in one color magnitude and about $7.2 \mathrm{~h}$ for MONS in an actual color. As the duration of an Earth twin transit is about $13 \mathrm{~h}$, these numbers are interesting, provided one neglects stellar activity. Detecting signals in the presence of this sort of non-white noise is complex, however.

The value of a transit candidate is limited without the ability to classify it. In the light of this analysis, it would be very practical for missions that intend to search for transiting terrestrial planets to include some way of obtaining color information. Otherwise, a smaller secondary mission would be required to obtain the necessary observations. Such an instrument would be of tremendous scientific value, not just for followingup transit candidates but also for any phenomena that involve small changes in intensity, such as asteroseismology.

Acknowledgements. I would like to thank the Danish Natural Sciences Research Council for financial support. I would also like to thank Hans Kjeldsen and Jørgen Christiansen-Dalsgaard for all the support they have provided me with over the past two years. I would also like to thank Torben Arentoft and Grzegorz Kopacki for putting up with my constant questions.

\section{References}

Abe, F., Bennett, D. P., Bond, I. A., et al. 2003, A\&A, 411, 493

Allen, C. W. 1976, Astrophysical Quantities, Third Ed. (University of London: The Athlone Press)

Baker, N. 1966, in Stellar Evolution, ed. R. F. Stein, \& A. G. W. Cameron (New York: Plenum), 333

Bessell, M. S. 1990, PASP, 102, 1181

Borucki, W. J., \& Sommers, A. L. 1984, Icarus, 58, 121

Brown, T. M., Charbonneau, D., Gilliland, R. L., Noyes, R. W., \& Burrows, A. 2001, ApJ, 552, 699

Charbonneau, D. [arXiv:astro-ph/0302216]

Christensen-Dalsgaard, J. 2002, in 1st Eddington Workshop on Stellar Structure and Habitiable Planet Finding, ed. F. Favata, I. W. Roxburgh, \& D. Galadi (Noordwijk: ESA Publications Divisiom), ESA SP- 485,25

Drake, A. J. 2003, ApJ, 589, 1020

Dreizler, S., Hauschildt, P. H., Kley, W., et al. 2003, A\&A, 402, 791

Gilliland, R. L., Brown, T. M., Kjeldsen, H., et al. 1993, AJ, 106, 2441

Konacki, M., Torres, G., Jha, S., \& Sasselov, D. D. 2003, ApJ, 597, 1076

Kotredes, L., Charbonneau, D., O’Donovan, F. T., \& Looper, D. L., http://www. astro.caltech.edu/ dc/

kotredes_sherlock_poster.pdf

Jenkins, J. M., \& Doyle, L. R. 2003, ApJ, 595, 429

Rosenblatt, F. 1971, Icarus, 14, 71

Seager, S., \& Mallén-Ornelas, G. 2003, ApJ, 585, 1038

Sung, Hwankyung, Bessell, M. S., Lee, Hee-Won, Kang, Yong Hee, \& Lee, See-Woo. 1999, MNRAS, 310, 982

Udalski, A., Paczynski, B., Zebrun, K., et al. 2002a, AcA, 52, 1

Udalski, A., Paczynski, B., Zebrun, K., et al. 2002b, AcA, 52, 115

Udalski, A., Szymanski, M., Kubiak, M., et al. 2002c, AcA, 52, 217 